\title{
Underwater Wideband Source Localization Using the Interference Pattern Matching
}

\author{
Seung-Yong Chun ${ }^{1}$, Se-Young Kim², and Ki-Man Kim² \\ ${ }^{1}$ Agency for Defense Development, \\ \#1 Hyun-dong, 645-016 Jinhae, Korea \\ ddoyong@dreamwiz.com \\ ${ }^{2}$ Dept. of Radio Communication Eng., Korea Maritime University, \\ \#1 Dongsam-dong, Yeongdo-ku, 606-791 Busan, Korea \\ sgtgfm@hhu.ac.kr, kimkim@hhu.ac.kr
}

\begin{abstract}
Recently many studies have been performed to detect underwater target by sensor network. This paper proposes an underwater source localization method based on wideband interference pattern matching. Matching of two interference patterns which seen in the sensor spectrograms, estimates a ratio of the range from source to two sensors according to the waveguide invariant theory and this ratio applied to circle of Apollonius. The circle of Apollonius is defined as the locus of all points whose distances from two fixed points are in a constant ratio so it is possible to represent the locus of potential source location. However, since ambiguity of absolute source location so it requires that additional equation which estimates another locus of the source. Therefore the hyperbola equation by estimating TDOA (Time Difference Of Arrival) is introduced into localization and finally cross point of two equations can be estimated as the source location. We performed simulation to test performance of the proposed localization method and then practiced error analysis of the results. And we tested performance of capability from a real-data collected during sea experiment. From simulation and experimental results, proposed algorithm represents that estimated position of target showed error of within $10 \%$.
\end{abstract}

Keywords: source localization, waveguide invariant, IPM (Interference Pattern Matching), circle of Apollonius, hyperbola equation.

\section{Introduction}

Source localization in shallow water has been considered many times over the past years and many studies have been achieved in order to improve the performance. In shallow water environment, complicated phenomenon such as multipath propagation and beam spreading degrades the performance of estimates the source. Conventional plane-wave beamforming and MUSIC (MUltiple SIgnal Classification) have been discussed general localization techniques. However these techniques dose not 
sufficiently consider that the propagation characteristics in ocean waveguide therefore in the real ocean their performance reduce with large localization error.

For this reason there are several attempts have been made to localize for a long range source by exploiting multi-modal dispersion such as MFP(Matched Field Processing) technique based on acoustic propagation model in multi-path environment[1], and recent array invariant theory derived by S.W. Lee[2].

In particular a study on localization technique using waveguide invariant theory has been attained. The range of the source can sometimes also be estimated by the much simpler waveguide invariant theory. The invariant parameter called $\beta$ is useful for describing the characteristic of the acoustic waveguide. However the waveguide invariant method requires knowledge of certain "invariant" parameter $\beta$ which unfortunately often vary with sound speed structure of the ocean. Recently several methods are introduced using the waveguide invariant theory and showed enhanced performance. But they are still dependent on the $\beta$ and ocean environment. So it is necessary to localize the source that independent of the $\beta$ without the information of the ocean environment. Therefore in this paper, we propose a source localization method that has advantages of not regard for $\beta$ and much simpler.

\section{Waveguide Invariant and Interference Pattern}

Interference pattern which seen in the sensor spectrograms collected from the moving ship-radiated noise arise from the mutual interference between modes reflected by the surface and the bottom. The slope of the interference pattern has been known invariant. Waveguide invariant parameter, designated as $\beta$, has been known that a slope of the interference pattern is directly proportional to the range of the source [3].

The $\beta$ is approximately 1 in the Pekeris waveguide however in the case of the real ocean the $\beta$ is variable with mode number, frequency and source depth, so knowledge of a certain invariant parameter is necessary to source localization.

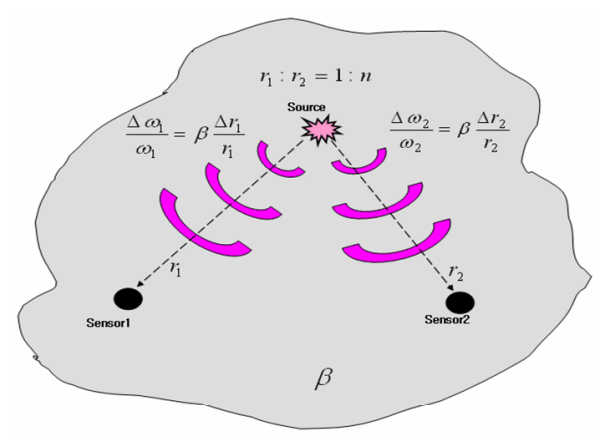

Fig. 1. The characteristic of waveguide invariant in identical pressure field 
If two sensors are used to source localization in identical acoustic propagation environment it is possible to detect the source without regard for $\beta$ because of the $\beta$ has identically effect on the each sensor. Figure 1 shows that the characteristics of waveguide invariant for two sensors which are located in identical pressure field.

The relationship between $\beta$ and slope of the interference pattern for each sensor can be expressed as

$$
\begin{aligned}
\frac{d \omega / \omega}{d r / r} & =\left(\frac{k_{m}-k_{n}}{\omega}\right) /\left(\frac{d k_{m}}{d \omega}-\frac{d k_{n}}{d \omega}\right) \\
& =-\left(\frac{1}{v_{m}}-\frac{1}{v_{n}}\right) /\left(\frac{1}{u_{m}}-\frac{1}{u_{n}}\right) \equiv \beta_{m n}
\end{aligned}
$$

Here $k_{m}$ and $k_{n}$ are $m$ th and $n$th mode wave number as a function of the frequency respectively, $v_{m}=\omega / k_{m}$ and $v_{n}=\omega / k_{n}$ are $m$ th and $n$th phase velocity respectively, $u_{m}=d \omega / d k_{m}$ and $u_{n}=d \omega / d k_{n}$ are $m$ th and $n$th group velocity respectively. Equation (1) summarized as follows

$$
\begin{aligned}
\frac{d \omega_{1}}{\omega_{1}} & =\beta \frac{d r_{1}}{r_{1}} \\
\frac{d \omega_{2}}{\omega_{2}} & =\beta \frac{d r_{2}}{r_{2}}
\end{aligned}
$$

$r_{1}$ and $\omega_{1}$ are the range from the source to sensor 1 and frequency of the interference pattern in spectrogram for sensor 1 , respectively. $r_{2}$ and $\omega_{2}$ are the range from the source to sensor 2 and frequency of interference pattern in spectrogram for sensor 2 , respectively. Since $\beta$ has identically effect on the each sensor equation (2) summarized as

$$
\frac{r_{1}}{r_{2}} \frac{\omega_{2}}{\omega}=\frac{d r_{1}}{d r_{2}} \frac{d \omega_{2}}{\omega}
$$

Assuming that $r_{1}=n r_{2}$ where $\mathrm{n}$ is ratio of the range from source to two sensors, one finds

$$
n=\frac{r_{2}}{r_{1}}=\frac{\omega_{2}}{\omega_{1}}
$$

From equation (4) it is notice that the ratio of frequency same as the ratio of range. Consequently ratio of the frequency between interference patterns which seen in each single sensor spectrogram, represents a ratio of range between source and each sensor. 


\section{Proposed Algorithm}

\subsection{Interference Pattern Matching (IPM)}

The ratio of the range between source and two sensors is estimated by interference pattern matching (IPM). Figure 2 shows a process of the IPM. Sensor 1 is selected reference spectrum and the spectrum of sensor 2 scaled until matched spectrum of sensor 1. If the RMSE (Root Mean Square Error) between sensor 1 spectrum and scaled sensor 2 spectrum is the most smaller then the ratio of scale can be estimated as the ratio of range.

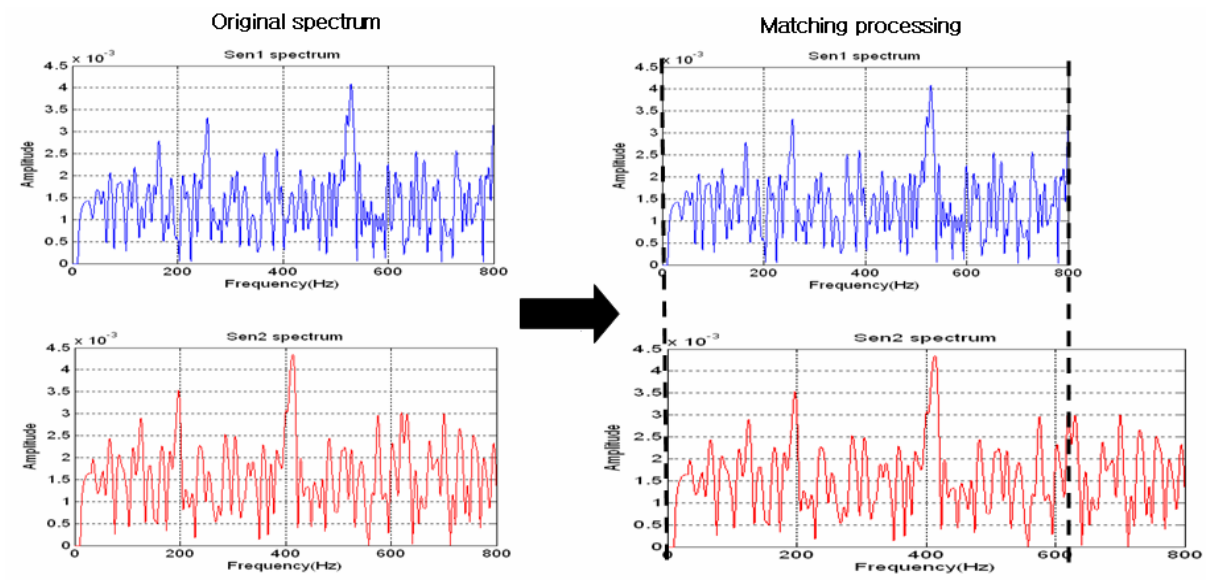

Fig. 2. Interference pattern matching process

Estimated ratio of range applied to circle of Apollonius. Circle of Apollonius shown in Figure 3 defined as the locus of a point whose distance from a fixed point is a multiple of its distance from another fixed point. If the multiple is equal to 1 , then the locus is a line which is perpendicular bisector of the segment of each fixed point and the multiple is not equal to 1, then it is a circle so called circle of Apollonius [6].

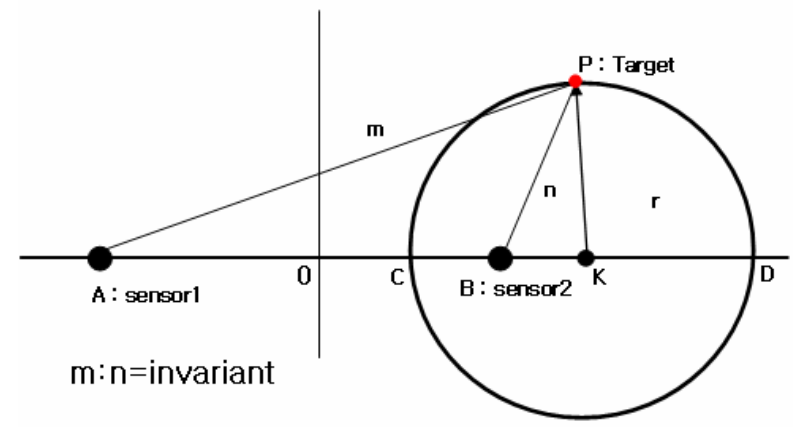

Fig. 3. Circle of Apollonius 


\subsection{Source Localization by Two Equations}

The result from IPM estimates the locus of the source using the circle equation derived from the principal of the circle of Apollonius. But it requires other equation in order to estimate the absolute source position because the circle equation can make only one of locus in the case of two sensors. So the second relative range between source and two sensors is estimated by introducing TDOA (Time Difference Of Arrival) technique [7]. The estimated TDOA between two sensors is applied to hyperbola equation. Finally cross point of the circle and hyperbola can be estimated as the position of the source. Figure 4 shows the interaction point of the circle of Apollonius and hyperbola.

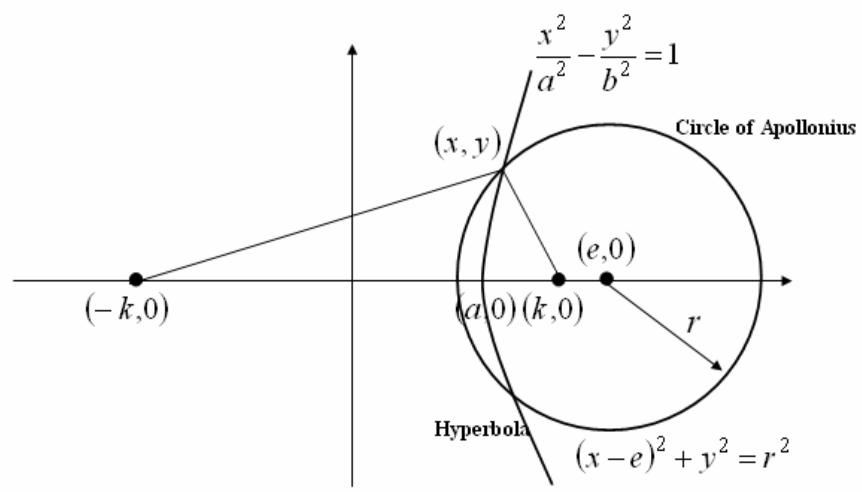

Fig. 4. Interaction point of the circle of Apollonius and hyperbola

The corresponding with coordinates of the source can be expressed as following solutions of two equations.

$$
\begin{aligned}
& x=\frac{a^{2} k+a \sqrt{a^{2} k^{2}-\left(a^{2}+b^{2}\right)\left(k^{2}-b^{2}-r^{2}\right)}}{a^{2}+b^{2}} \\
& y= \pm \sqrt{\frac{b^{2}}{a^{2}} k^{2}-b^{2}}, \text { if } x^{2}>a^{2}
\end{aligned}
$$

\section{Simulation and Experimental Results}

We performed simulation of 3 scenarios to test the IPM algorithm and proposed localization method and then practiced error analysis of the results. And we tested performance of a real-data collected during MAPLE-05 experiment applied to the proposed algorithm. 


\subsection{Simulation Results}

We demonstrate the performance of the proposed localization algorithm with simulated pressure field based on KRAKEN normal mode program [8]. Figure 5 shows the three cases for the simulation.

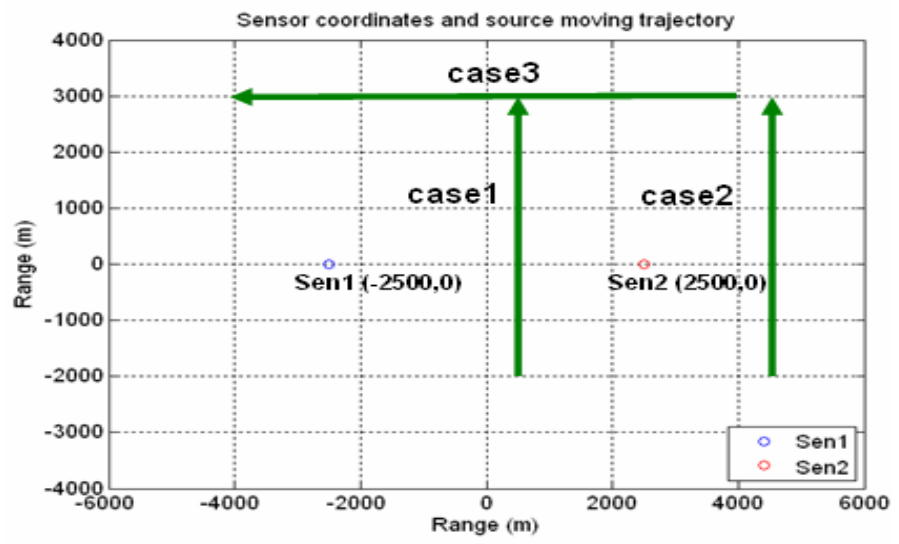

Fig. 5. Three cases for the simulation

The distance of each sensor is $5 \mathrm{~km}$. The speed of moving target is 5 knot and range of trajectory is $5 \mathrm{~km}$ during about 32 minutes for case 1 and case 2 . In case 3 range of trajectory is $8 \mathrm{~km}$ during about 51 minutes. Target signal has 1 to $800 \mathrm{~Hz}$ band. The source localization performed at intervals of $250 \mathrm{~m}$ in trajectory and the results of error for simulation are presented at table 1 .

Table 1. Results of error in simulation

\begin{tabular}{|l|c|c|c|}
\hline & IPM error & Mean tracking error(m) & Mean tracking error rates (\%) \\
\hline Case1 & 0.0031 & 47.37 & 4.14 \\
\hline Case2 & 0.1787 & 210.87 & 4.39 \\
\hline Case3 & 0.0133 & 96.91 & 2.46 \\
\hline
\end{tabular}

From table 1, the proposed algorithm based on IPM seemed to have excellent performance whose mean tracking error rates is within 5\%, but if examine a RMSE (Roots Mean Square Error) pattern according to the adjust IPM ratio, it showed the minimum value even in error point. We conclude this is due to the sensor spectrum adjusted linearly without previous knowledge of environment on $\beta$ and environmental parameter of the real ocean. In particular results of case 2 represent a great tracking 
error proportional to IPM error. So in order to improve the performance it is necessary to estimate correct ratio of IPM.

\subsection{Experimental Results}

The source tracking result for experiment demonstrated at figure 6 . The speed of moving target is 4 knot and the distance of each sensor is $66 \mathrm{~m}$. Target signal has 1 to $400 \mathrm{~Hz}$ band. From the result though it is note that a little difference between true position and estimated position but tracking tend to true trajectory.

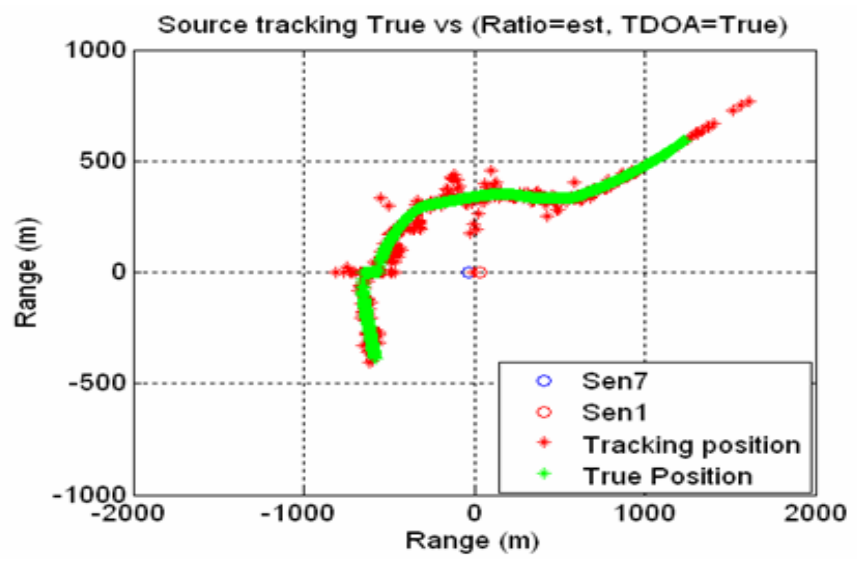

Fig. 6. Source tracking result for MAPLE 05 experiment

If examine the experimental result, since the estimated position of target showed error of within $10 \%$, so proposed algorithm is even available in real ocean. The IPM proposed in this paper and localization method with TDOA are possible to be applied to identical pressure field without regard for $\beta$, and need no previous knowledge of environment. Also, because only two sensors make localization possible, this is expected to adapt sufficiently to the field of active sonar and passive sonar using property of broadband signal.

\section{Conclusions}

The localization method based on IPM has been introduced for estimates source position without regard for $\beta$ and knowledge of the ocean environment. It has been shown that proposed method does not require extensive computations because only two equations are used. The ability to make simple and accurate source tracking by the proposed method has been demonstrated with simulation and real data from the MAPLE 05. In future work, it is needs to test the performance in range dependent environment and the research of 3-D source localization. 


\section{References}

1. Baggeroer, A.B., Kuperman, W.A., Schmidt, H.: Matched field processing: source localization in correlated as an optimum parameter estimation problem. J. Acoust. Soc. Am. 83, 571-587 (1988)

2. Lee, S.W., Makis, N.C.: The array invariant. J. Acoust. Soc. Am. 119(1), 336-351 (2006)

3. D'Spain, G.L., Kuperman, W.A.: Application of waveguide invariants to analysis of spectrograms from shallow environments that vary in range and azimuth. J. Acoust. Soc. Am. 106(5), 2454-2468 (1999)

4. Brekhovskikh, L.M., Lysanov, Y.: Fundamentals of Ocean Acoustics, 3rd edn. Springer, Heidelberg (2003)

5. Jensen, F.B., Kuperman, W.A., Porter, M.B., Schmidt, H.: Computational Ocean Acoustics. AIP, New York (1994)

6. Georgia University dept. of mathematics education, http://jwilson.coe.uga.edu

7. Fertner, A., Sjolund, A.: Comparison of various time delay estimation methods by computer simulation. IEEE Trans. Acoust. Speech Signal Process. ASSP-34(5), 1329-1330 (1986)

8. Porter, M.B.: The KRAKEN Normal Mode program, SACLANT Undersea Research Centre (1994) 